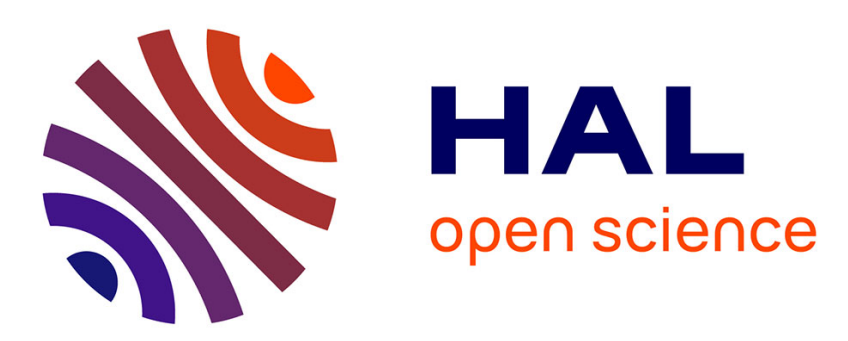

\title{
Recent advances on yield stress and elasticity of fresh cement-based materials
}

Nicolas Roussel, Hela Bessaies Bey, S. Kawashima, D. Marchon, K. Vasilic, R. Wolfs

\section{- To cite this version:}

Nicolas Roussel, Hela Bessaies Bey, S. Kawashima, D. Marchon, K. Vasilic, et al.. Recent advances on yield stress and elasticity of fresh cement-based materials. Cement and Concrete Research, 2019, 124, 11 p. 10.1016/j.cemconres.2019.105798 . hal-02494476

\section{HAL Id: hal-02494476 https://hal.science/hal-02494476}

Submitted on 25 May 2021

HAL is a multi-disciplinary open access archive for the deposit and dissemination of scientific research documents, whether they are published or not. The documents may come from teaching and research institutions in France or abroad, or from public or private research centers.
L'archive ouverte pluridisciplinaire HAL, est destinée au dépôt et à la diffusion de documents scientifiques de niveau recherche, publiés ou non, émanant des établissements d'enseignement et de recherche français ou étrangers, des laboratoires publics ou privés. 


\section{Recent advances on yield stress and elasticity of fresh cement- 2 based materials.}

3

4 Roussel, N. ${ }^{1}$, Bessaies-Bey ${ }^{1}, \mathrm{H}$, Kawashima, S. ${ }^{2}$, Marchon; $D .^{3}$, Vasilic, K. ${ }^{4}$, Wolfs, $R .{ }^{5}$ 5

$6{ }^{1}$ IFSTTAR, East Paris University, France

72 Department of Civil Engineering and Engineering Mechanics, Columbia University, New

8 York, NY, United States

$9{ }^{3}$ Department of Civil and Environmental Engineering, University of California, Berkeley, 10 CA, United States.

$11{ }^{4}$ German Society for Concrete and Construction Technology (DBV), Berlin, Germany.

$12{ }^{5}$ Department of the Built Environment, Eindhoven University of Technology, Eindhoven, 13 The Netherlands.

\section{Abstract}

16 The interest in the elastic and yielding properties of fresh cement-based materials has 17 recently grown due to the development of new processing techniques, which avoid the 18 use of standard formworks. Without support, the material shaping relies only on the 19 mechanical properties of the fresh material. Within this frame, the point of this paper is 20 to gather the accepted knowledge along with the most recent advances on both yield 21 stress and elasticity of fresh cement- base materials. In the first part, we will go through 22 the physical and chemical origin of these macroscopic properties and their evolutions. In 23 the second part, we will describe the way they can be measured while the third part will 24 deal with the way they can be controlled and tuned. Finally, the most recent models for 25 both properties prediction from mix design and processing modeling from rheological 26 measurements will be described.

27 


\section{Introduction}

2 The rheological behavior of fresh cement-based materials has been an important field of

3 research for decades. It has, however, over the years primarily focused on the way fresh

4 cement based materials flow. As concrete, mortar or grouts processing mostly involves

5 sub-processes such as mixing, pumping, spraying and pouring, non-Newtonian fluids

6 mechanics was considered to be the sub-field of mechanical sciences that could improve

7 our understanding of fresh cement-based materials processing.

8 The elasto-plastic behavior and the material ductility were therefore features that were

9 considered to belong to the set of mechanical properties that only hardened concrete

10 was concerned with and fresh cement-based materials were classified as non-

11 Newtonian granular fluids.

12 It is interesting enough to note that beginning of setting was, within this frame,

13 historically defined as the time required for the material to display an elastic modulus. It

14 was however shown that fresh cement-based materials do display an elastic behavior

15 for observations times as short as a few seconds after the end of mixing (L. Nachbauch

16 et al., 2001) (N. Roussel et al., 2012). After such short time scales, the percolated

17 network of interacting particles indeed display an elastic modulus of the order of several

18 hundreds of $\mathrm{Pa}$ and a strength of the order of a few tens of $\mathrm{Pa} .28$ days and an extremely

19 complex hydration reaction later, this strength has turned into several tens of MPa. As a

20 force sensor able to measure a strength variation over 6 orders of magnitude does not

21 exist, beginning of setting was therefore defined as the time required for the material to

22 display an elastic modulus that was measurable using a standard concrete compression

23 testing machine (i.e. unable to measure the low values of elastic modulus and strength

24 typical of fresh materials). This contributed into strengthening the idea that fresh-

25 cement based materials were pasty fluids with neglectable elasticity.

26 It has however to be acknowledged that, until recently, the knowledge of the elastic

27 behavior of fresh cement-based materials was of little interest as they were supposed to

28 flow from the mixer to the formwork, fill it properly under the sole effect of gravity and

29 set. In order to ensure that the material properly fills the formwork along with the 
1 centimetric porosity between the reinforcing steel bars, high energy vibrating pokers

2 (vibration amplitude of the order of a couple mms for frequencies of the order of couple

3 hundreds $\mathrm{Hz}$ ) were used to apply accelerations roughly ten times higher than the natural

4 gravity acceleration to the fresh material. At such a level of stress, any interactions or

5 elastic inter-particle network is destroyed and the material behaves indeed as a non-

6 Newtonian liquid.

7 Only recently, with the increased industrial use of extremely fluid cement-based

8 materials such as self-compacting or self-leveling mortars, concretes or floor screeds,

9 elasticity and yield stress started to matter. Without vibration, the filling degree or the

10 shape conformity was shown to depend on the competition between gravity and yield

11 stress (N. Roussel, 2007a) whereas some features such as pressure formwork were

12 related to the elasticity and structuration (i.e. the increase in elastic modulus or yield

13 stress with resting time) of the fresh material (P. Billberg, 2003), (G. Orvarlez et al.,

14 2007).

15 Even more recently, the development of 3D printing technologies for and by the 16 concrete industry stressed the need for a better assessment and understanding of the 17 elastic and yield properties of fresh cement-based materials. Most 3D printing 18 applications and demonstrators are based on extrusion, where a cement-based material 19 is extruded to form sequential layers via a digitally controlled nozzle mounted on a 20 robot arm, gantry or crane. The processes are similar to conventional Additive 21 Manufacturing processes except that the material and scale of manufacture generate 22 unique challenges and questions: the formulation of mixes that are pumpable, yet stable 23 in shape once placed without formwork; the production of reliable interlayer bonding; 24 and how to control the geometry of printed parts within appropriate geometrical 25 tolerances. All these questions relate to the yield stress and elasticity of fresh cement26 based materials.

27 Within such a frame, the point of this paper is to gather the accepted knowledge along 28 with the most recent advances on these features. In the first part, we will go through 29 the physical and chemical origin of these macroscopic properties in the fresh state and 
1 their evolutions. In the second part, we will describe the way they can be measured

2 while the third part will deal with the way they can be controlled and tuned. Finally, the

3 most recent models for both properties prediction from mix design and process

4 modeling from rheological measurements will be described.

2. Yield stress and elasticity in fresh cement-based materials: physical origin

\subsection{Brownian motion, Van der Waals forces and buoyancy}

8 It is now accepted that the dominant interactions in a fresh cement paste are the

9 following: colloidal attractive forces, gravity forces and Brownian effects (N. Roussel et 10 al., 2010). Hydrodynamic forces (including laminar viscous dissipation, particle kinetic 11 energy and lubrication) only occurs when the system is sheared and flows. Note that, 12 once mixing is over and the interstitial fluid is forming a continuous phase, capillary 13 forces in cement-based systems can be neglected.

14 The main competition in the system at rest involves therefore colloidal attractive forces, 15 Brownian motion and gravity forces. This competition was reported to result in one of 16 the following regimes (A. Perrot et al., 2012):

17 If colloidal attractive forces dominate Brownian motion, which is the case for most cementitious materials in industry, a percolated network of interacting particles appears

19 within the suspension. It is able to withstand an external stress up to a critical value 20 called yield stress. Because it originates from the attractive van der Waals forces (Flatt, 21 R.J. et al., 2004), this yield stress scales with the inverse power two of the inter-particle 22 separating distance and is therefore strongly dependent on the presence of adsorbed 23 polymers at pseudo-contact points between particles (see section 3). This percolated 24 interaction network has a rigidity, which dictates in turn the material elasticity 25 (Nachbauch et al., 2001); (W.G. Lei and L.J. Struble, 1997), (N. Roussel et al., 2012).

26 If Brownian motion dominates colloidal attractive forces (in the case of diluted systems 27 or systems at high admixture concentration (see section 3)), the suspension does not 28 have any yield stress. The network of attractive colloidal interactions is instantaneously 29 destroyed by Brownian agitation and is not able to withstand any external stress. In this 
1 particular case, Brownian motion could dominate gravity forces. The result would be a

2 stable Newtonian viscous suspension. However, according to the order of magnitudes of

3 the two phenomena, this cannot happen in the case of typical cementitious materials.

4 Suspensions, in which Brownian motion dominates colloidal forces, shall therefore

5 always be unstable and display high levels of sedimentation (N. Roussel et al., 2010), (N.

6 Massoussi et al., 2017).

7 If colloidal attractive forces dominate gravity forces, particles are trapped in the

8 colloidal interactions network and are therefore not able to rearrange their relative

9 positions. The suspension is stable. There is neither bleeding nor sedimentation and the

10 fresh paste stays homogeneous.

11 If gravity forces dominate colloidal attractive forces, particles may settle. This induces a 12 relative displacement of the cement particles within the interstitial fluid or a relative 13 displacement of the interstitial fluid between the cement particles. This phenomenon 14 called "bleeding" in practice is time driven and is influenced by the viscosity of the 15 interstitial fluid and the permeability of the porous medium formed by the interacting 16 cement grains (which mainly depends on the solid volume fraction and on the average 17 diameter of the grains (Picandet et al., 2011)). It was shown recently that bleeding 18 relates to the formation of preferred water extraction channels resulting from a local 19 and partial rearrangement of the cement particles configuration (N. Massoussi et al., 20 2017). As such, it is a heterogeneous process in contrast to the former approach in 21 literature describing bleeding as a homogeneous consolidation phenomenon (L. 22 Josserand et al., 2006). Within very specific conditions, bleeding may be adequately 23 limited by the above mix design factors. It is in this regime only that fluid cement pastes 24 allowing for the production of fluid concrete can be mix designed.

\subsection{Hydrates nucleation, thixotropy and structuration}

27 Casting of concrete occurs within the induction period, which is generally considered to 28 be a period of little chemical activity. However, there are still the effects of cement 29 hydration, where early hydrates form and subsequently impact the workability or 
1 rheology of the fresh suspension. This has been more precisely tied to $\mathrm{C}-\mathrm{S}-\mathrm{H}$ nucleation

2 in both cement (Lei and Struble 1997) and tricalcium silicate (L. Nachbaur et al. 2001)

3 systems, and more recently supported by (N. Roussel et al. 2012) through a description

4 of structuration and thixotropy. In this latter study, the general behavior of fresh

5 cement-based systems at rest was described, and it is again presented here in Fig. 1

6 through yield stress evolution and a microstructural view of cement interaction and C-S-

$7 \mathrm{H}$ nucleation and growth. Immediately after mixing, the cement particles are in a

8 dispersed state, where the degree of dispersion is dependent on mixing energy (Fig. 1a).

9 Once at rest, the non-contact, colloidal interactions discussed in Sec 2.1 lead to early

10 (few sec) flocculation of the fresh suspension (Fig. 1b). From there, C-S-H nucleation

11 occurs and forms bridges between cement particles/flocs (Fig. 1c), and gives rise to an

12 increasingly interconnected, rigid structure through continued $\mathrm{C}-\mathrm{S}-\mathrm{H}$ nucleation and

13 growth (Fig. 1d). This structure underlies increase in yield stress (flow onset) and

14 elasticity over time.

15 Thixotropy - structural breakdown and build-up - is theoretically a completely 16 reversible phenomenon. However, this is not the case for cement-based materials due 17 to the continuous progression of hydration. Cement thixotropy arises from a 18 combination of general flocculation/deflocculation behavior, as well as 19 formation/breakage of C-S-H bridges (J.E. Wallevik 2009) (N. Roussel et al. 2012). If 20 there is sufficient shearing energy (i.e. mixing, pumping, casting), the aforementioned C$21 \mathrm{~S}-\mathrm{H}$ bridges can be broken down and flow can be restored. At the same time, some C-S$22 \mathrm{H}$ bridges will be irreversible under the given shearing conditions and a corresponding 23 level of structure will remain. Over time, the presence of irreversible C-S-H bridges will 24 increase (in size and frequency) (Jiang et al., 1995), (A. Nonat et al., 1997), which gives 25 rise to an increase in yield stress associated with flow onset.

26 Role of hydration on workability has been of practical significance for workability loss, 27 where mixing/shearing can no longer restore flowability. And thixotropy has been of 28 practical significance for select applications, e.g. SCC formwork pressure where it is now 29 well accepted that structural build-up behavior of the fresh SCC has a direct effect on 
1 lateral pressure exerted on the formwork wall [G. Ovarlez and N. Roussel 2006), (J.

2 Assaad et al. 2003a), (J. Assaad et al. 2003b). More recently, the connection between

3 structural build-up behavior and C-S-H nucleation has been shown (T. Lecompte and A.

4 Perrot, 2017) (Ma et al., 2018), (D. Lowke 2018). The role of C-S-H nucleation on

5 structural build-up kinetics (both flow onset and elasticity) becomes critically more

6 important for AM, as recently summarized by (L. Reiter et al 2018). Short-term kinetics

7 are important in controlling material shape stability immediately after deposition, while

8 long-term kinetics (approaching setting) is important for determining building rate. It

9 has been shown that yield stress evolution goes from linear to nonlinear over longer

10 periods (A. Perrot et al., 2015), which has more recently been tied to increase in solid

11 volume fraction and decrease in packing fraction by C-S-H nucleation (T. Lecompte and

12 A. Perrot, 2017). This linear to non-linear yield stress evolution has been incorporated

13 into a theoretical framework to predict building rate for AM (A. Perrot et al., 2015). It

14 can finally be noted that the behavior of the material becomes closer to the one of a

15 brittle solid and further from the one of a plastic paste (Mettler et al., 2016).

\subsection{From cement paste to mortars and concretes}

Adding particles such as sand and/or coarse aggregates to a cement paste to produce a mortar or a concrete can conceptually be considered as adding rigid inert non-Brownian non-colloidal particles into a yield stress fluid as long as the typical size of the constitutive elements of the paste (i.e. cement grains of average size of 10 micrometers) can be neglected in front of the typical size of the particles (i.e. sand or coarse aggregates of respective average size of 1 or $10 \mathrm{~mm}$ ). In this specific case, the rheological properties of the suspension shall depend only on the rheological properties of the suspending fluid (as long as the presence of the particles do not affect it) and on the particle volume fraction, shape and size distribution. With the aim of providing such generic results, (F. Mahaut et al., 2008a) have performed an experimental study on a broad range of materials. They have suspended beads of various sizes and made of various materials in very different pastes whose common point was to exhibit a yield 
1 stress. They showed that the dimensionless yield stress of such mono-disperse

2 suspensions indeed relates to the particle volume fraction only. It should be noted that

3 this should apply to any homogeneous and isotropic suspension of particles in a yield

4 stress fluid as long as the particles have only mechanical interactions with the paste (i.e.

5 when there are no specific physicochemical interactions between the particles, and

6 between the particles and the paste). The same authors have moreover studied

7 suspensions of coarse spherical particles in a cement paste and demonstrated that they

8 behave exactly as any other suspension once all artifacts have been ruled out by

9 adequate experimental protocols (F. Mahaut et al., 2008b). All the above studies

10 emphasize the fundamental role played by the packing properties of the inclusions

11 added to the cement paste (J. Yammine et al., 2008).

12 From a thixotropy point of view, it was also shown that it is sufficient to know how the

13 interstitial cement paste evolves in time to predict the mortar or concrete evolution

14 (assuming there is no water absorption). As a consequence, if the mechanical impact of

15 the non-colloidal particles is to increase the yield stress by a given factor, then their

16 impact on the structuration rate of the paste is to increase by the same factor. It is thus

17 sufficient to measure the cement paste yield stress evolution in time and to measure

18 the increase of yield stress with volume fraction for a single resting time to infer the

19 value of the structuration rate of the mortar or concrete at any time ( $F$. Mahaut et al., 20 2008b).

3. Yield stress and elasticity in fresh cement-based materials: measurements

\subsection{Industrial test for yield stress measurements}

Regardless of the exact standards or geometry of slump, slump flow, L-Box or J-ring tests, the following general procedure is common for these tests: a mold or reservoir of

26 a given standard shape or volume is filled with the cementitious material to be tested.

27 The material is poured, a mold is lifted or a gate is opened and flow occurs if the shear

28 stress generated by gravity inside the sample is higher than the yield stress of the

29 material. As the material is flowing, the thickness of the sample and, therefore, the 
1 stress generated by gravity decrease. If the stress in the tested sample becomes lower

2 than the yield stress of the tested material, flow stops. The shape at stoppage is

3 therefore directly linked to the yield stress of the tested material if inertia or surface

4 tension effects can be neglected. Several correlations between the measured

5 geometrical quantities such as slump flow and slump and yield stress can be found in

6 literature (J. Murata, 1984), (N. Pashias et al., 1996), (Schowalter, 1998), (S. Clayton et

7 al., 2003), (A.W. Saak et al., 2004) (W.R. Schowalter and G. Christensen, 1998) (N.

8 Roussel and P. Coussot, 2005), (N. Roussel et al. 2005) (N. Roussel, 2006), (N. Roussel,

9 2007) (T.L.H. Nguyen et al., 2006).

3.2. Rheometry: flow onset and critical strain measurements

12 When the flow onset and critical strain of fresh cement-based materials are of interest,

13 a typical measurement consists of using a rheometer and applying a constant low shear

14 rate, starting from rest, and then observing the characteristic stress peak and critical

15 strain associated with flow onset. However, as these materials are thixotropic, their

16 rheological behavior depends on the flow history and their characterization requires a

17 rigorous procedure to ensure reproducibility. Before starting the measurement, the

18 suspension has to be pre-sheared to ensure that all tests start from the same destructed

19 state (D. Feys et al, 2017). Moreover, in order to study in detail the evolution of shear

20 stress over a large range of strains, a compromise must be reached between the

21 duration of the test and its accuracy.

22 A specific measurement protocol has been developed by (N. Roussel et al., 2012) for 23 cement suspensions. First, the sample is pre-sheared at $150 \mathrm{~s}^{-1}$ for $200 \mathrm{~s}$. After 5 minutes

24 of resting time, a constant shear rate of $0.005 \mathrm{~s}^{-1}$ is applied to the sample.

25 This protocol allows for the measurement of a linear increase of shear stress 26 corresponding to the elastic behavior of the material in its solid regime. Above a critical

27 strain around a few \% and when shear stress reaches yield stress, the material starts to

28 yield and flow (see Fig. 3a). This stress peak is associated to the breakage of the network

29 of attractive colloidal interactions between cement particles. At a critical shear strain of 
1 the order of few hundredth of \%, another peak can be identified and is associated to the

2 breakage of the hydrates formed between flocculated cement grains (see Fig. 3b).

\subsection{Rheometry: oscillations}

5 Small amplitude oscillatory shear (SAOS) is a common way to probe the viscoelastic

6 properties of soft materials, and it has been found to be suitable for fresh cement

7 pastes to study hydration and stiffening behavior (or what we now term structuration)

8 [A. Papo and B. Caufin, 1991), (P.Banfill et al. 1991), (Schultz et al., 1993); (Z. Sun et al.

9 2006). In SAOS, a small sinusoidal strain is applied to the material to obtain a stress

10 response. The storage modulus $\left(G^{\prime}\right)$ is the in-phase, elastic component of the response

11 while the loss modulus (G") is the out-of-phase, viscous component. The complex

12 modulus $\left(G^{*}=G^{\prime}+G^{\prime \prime} i\right)$ and phase lag provide further description of viscoelasticity.

13 Through performing a strain sweep, i.e. progressively increasing amplitude, we can

14 obtain a measure of critical strain, which marks the end of the linear viscoelastic regime

15 (LVR) - theoretically the limit of the material's ability to respond elastically and keep its

16 structure intact. Within the LVR, G' remains constant and independent of applied strain.

17 If the critical strain is exceeded, there is a significant drop in $\mathrm{G}^{\prime}$, indicating the structure

18 has been irreversibly broken. A frequency of $1 \mathrm{~Hz}$ is commonly used for cement pastes

19 and found to be within the LVR. And the critical strain is typically found to be in the

20 order of $10^{-4}$, which corresponds to the scale of C-S-H bridges that form between

21 cement particles during the early stages of hydration (N. Roussel et al. 2012). Therefore,

22 SAOS can be used as a tool to probe the rigid structure formed progressively through C-

$23 \mathrm{~S}-\mathrm{H}$ nucleation and growth, discussed in Sec 2.2.

24 By applying a sinusoidal strain within the LVR, we can obtain a continuous measure of 25 structural build-up of a material at rest. For cement-based systems, it is well accepted 26 that monitoring $G^{\prime}$ provides a measure of structure due to rigidification and, since the 27 material is probed within the LVR, resultant elasticity. Further, G' is related to 28 instantaneous (or apparent) shear modulus, which is the slope of the shear stress-strain 29 curve obtained by applying a sufficiently low shear rate. Importantly, it should be noted 
1 that short-term (few sec) increase in $G^{\prime}$ is tied to colloidal interactions, while long-term

2 linear increase in $\mathrm{G}^{\prime}$ is due to C-S-H nucleation (N. Roussel et al., 2012). This has more

3 recently been supported by (A.M. Mostafa and A. Yahia, 2016) in comparing G' and

4 phase angle evolution between cement versus model calcium carbonate suspensions -

5 in the latter there was a short-term increase in $G^{\prime}$ and decrease in phase lag due to

6 percolation, and no long-term increase due to the absence of hydration and

7 corresponding rigidification. A physico-chemical model has been proposed that can

8 predict structural build-up due to colloidal interactions and chemical rigidification based

9 on mix parameters (A.M. Mostafa and A. Yahia, 2017).

10 It has been shown that increase in $G^{\prime}$ does not necessarily coincide with an increase in

11 static yield stress due to differing mechanisms (S. Ma et al., 2018), i.e. G' arises from C-S-

$12 \mathrm{H}$ bridges while yield stress arises from a combination of colloidal interactions and $\mathrm{C}-\mathrm{S}-\mathrm{H}$

13 bridges that remain unbroken at higher applied strains. However, since C-S-H nucleation

14 is an overlapping mechanism, these parameters have also been found to be similar (Q.

15 Yuan et al., 2017). In evaluating structuration, it is therefore necessary to measure both

16 yield stress (through flow onset) and elasticity (through oscillations). This becomes

17 particularly important for AM, as we are interested in the contribution of both colloidal

18 and hydration effects on the development of the undisturbed structure for controlling

19 shape stability of the layer, building rate, and ultimately buckling stability of the layered

20 structure. It should be noted that the potential of large amplitude oscillatory shear to

21 characterize fresh cement pastes has recently been demonstrated (T. Conte and M.

22 Chaouche, 2016). Although it can be a powerful characterization tool for evaluating

23 thixotropy, its suitability for cements and quantitative interpretation of data needs

24 further exploration.

4. Yield stress in fresh cement-based materials: effects of admixtures

4.1. Repulsive inter-particle forces and decrease in yield stress

28 Several types of admixtures can decrease the strength of the flocculated network of 29 cement particles in a paste by generating repulsive forces between particles and 
1 increasing therefore the average inter-particle distance (see section 2.1) (J. Gelardi et

2 al., 2015). For instance, when adsorbed on cement surfaces, concrete dispersants can

3 provide electrostatic and/or steric repulsion between particles. The most efficient and

4 famous ones are the comb-shaped polycarboxylate ether (PCE) super-plasticizers that

5 have a negatively charged backbone adsorbing on particles surface and non-adsorbing

6 side chains inducing steric hindrance (J. Gelardi et al. 2015), (R.J. Flatt and Schober,

7 2012).

8 At equilibrium, adsorbed macromolecules have a preferred conformation of adsorption

9 on the surface of cement particles (R.J. Flatt et al., 2009). As soon as two covered

10 particles approach and the layers of polymer overlap, a repulsive steric force occurs

11 between the particles. This comes from a change of adsorption conformation of the

12 polymer, which results in a loss of entropy, together with a rise of osmotic pressure

13 coming from an increase of the concentration of polymer between the two approaching

14 particles, which prevents them from approaching further (J. Gelardi and R.J. Flatt, 2015).

15 The coverage degree and the thickness of the layer of adsorbed dispersants are

16 therefore of great importance as they dictate the minimum distance between two

17 particles before repulsion and impose therefore the value of the attractive Van der

18 Waals forces. This means that they rule the dispersion ability and therefore yield stress

19 and elasticity. They also depend on the dosage of polymer and on the surface occupied

20 by one molecule, which is itself dependent on the molecular structure of the polymer

21 (R.J. Flatt et al., 2009), (J. Gelardi and R.J. Flatt, 2015).

\subsection{Attractive inter-particle forces and increase in yield stress}

\section{Bridging forces}

25 Bridging forces arise when a polymer chain adsorbs simultaneously at the surface of two

26 or more particles. For bridging to occur, the polymer chain must be long enough to

27 reach the surface of at least two particles and must have an affinity with the surface

28 particle. It was shown by [Tadros et al., 2012] that an optimal bridging flocculation is

29 attained at half particles surface coverage. At higher concentrations, an adsorbed 
1 polymer on one particle might not find an empty site on another particle. In cement

2 based materials, most viscosity modifying admixtures (VMA) are able to bridge cement

3 particles, which increases the macroscopic yield stress of the suspension to a certain

4 extent (C. Brumaud et al., 2014), (H. Bessaies-Bey et al.,2015) (see Fig. 4). Some VMAs

5 also strongly modify the critical strain of cement paste. By comparing the flow onset of

6 the reference cement paste and cement pastes containing polyacrylamide and cellulose

7 ethers, we note that cellulose ether were found to allow for the mix design of softer

8 systems (i.e. lower elastic modulus) in comparison with the reference cement paste and

9 the cement containing polyacrylamide (C. Brumaud et al., 2014), (H. Bessaies-Bey et al.,

10 2015]. On the other hand, pastes containing polyacrylamide were found to exhibit a

11 higher elastic modulus. Such organic admixtures are essential for promoting particle

12 flocculation for shotcrete applications, mono-layer render mortar or tile adhesives (C.

13 Brumaud et al., 2011) along with additive manufacturing of concrete (D. Marchon et al., 14 2018).

\section{Attractive depletion forces}

17 Fig. 5 shows the evolution of a cement paste yield stress as a function of PCE dosage. In 18 the first regime (i.e. low dosages), we note a decrease in yield stress as PCE dosage 19 increases. However, above a critical concentration, the latter trend is inverted and an 20 increase in PCE dosage leads to an increase in yield stress. For this PCE and in this 21 dosage range, cement particle surfaces are fully covered by adsorbed PCE and any 22 additional PCE remains in the suspending fluid. These results suggest that excess in 23 super-plasticizer may be at the origin of attractive depletion forces, which causes a 24 fluidity loss.

25 It is now accepted in the literature of colloidal particles that the addition of non26 adsorbing polymer to a suspension can generate depletion attractive forces, which may 27 cause particle flocculation (S. Asakura et al., 1954), (S. Asakura et al., 1958). When a 28 polymer has no affinity with a surface it is excluded leading to a formation of a region of 29 pure solvent. The differences in osmotic pressure between the polymer depleted (or 
1 pure solvent) region and the bulk polymer solution lead to an attraction between the

2 particles. In cement based materials, (H. Bessaies-bey et al, 2018) showed recently that

3 non-adsorbed polymers induce attractive depletion forces, which may lead to an

4 increase in cement paste yield stress. These forces mainly depend on the polymer

5 concentration rather than their molecular weight.

6 It should be kept in mind that, in the absence of super-plasticizers, Van der Waals

7 attractive forces dominate depletion forces and the consequence of non-adsorbing

8 polymers on yield stress is negligible. However, in the presence of super-plasticizers, the

9 magnitude of the attractive van der Waals forces decreases (see section 4.1) and

10 attractive depletion forces induced by non-adsorbing polymers become important and

11 greatly contribute to yield stress and elasticity.

12

\section{Hydrophobic forces}

14 Although surfactant molecules are mainly incorporated in cement based materials to

15 stabilize aqueous foams, it was recently shown by (B. Feneuil et al., 2017) that these

16 molecules can adsorb on the surface of cement particles and introduce attractive

17 hydrophobic forces in the system.

18 When adsorbed at the surface of cement particles, surfactants can turn the surface

19 hydrophobic. As hydrophobic surfaces tend to attract each other (J. Israelachvili et al.,

20 1984), (E. Meyer et al., 2006), the suspension flocculates and the yield stress increases.

21 It should be kept in mind that, above a critical concentration of surfactant in solution,

22 micelles may form and affect the mechanism of action of the surfactant.

\subsection{Competitive adsorption between admixtures}

Modern cement-based materials tend to include more than one type of admixture in

26 order to achieve required rheological properties. However, the blending of these

27 chemical admixtures has to be well controlled to limit incompatibilities and to achieve

28 the intended effect. As shown in the above sections, the mechanism of action of most

29 chemical admixtures is very sensitive to their adsorption on the surface of cement 
1 particles. The blending of these chemical admixtures can generate a competitive

2 adsorption on particles surface, which will, in turn, influence their performances. Many

3 examples of competitive adsorption between a superplasticizer and sulfate ions ( $\mathrm{K}$.

4 Yamada et al., 2001), (R.J. Flatt et al., 2009], hydroxides (R.J. Flatt et al., 2015), (D.

5 Marchon et al., 2013), citrates (J. Plank et al., 2008) and other organic admixtures (J.

6 Plank et al., 2007), (J. Plank et al., 2010), (H. Bessaies-Bey et al., 2016) have been studied

7 in literature. By occupying the adsorbing sites at the surface of cement particles, so-

8 called "displacers" restrict the adsorption of super-plasticizer and considerably affect

9 their dispersion efficiency as shown in Error! Reference source not found.6 in presence

10 of sodium gluconate. Depending on the industrial application, competitive adsorption

11 can also be an advantage leading to synergetic effects between adsorbing species.

12 Indeed, it was observed in (H. Bessaies-Bey et al., 2016) that a super-plasticizer can

13 inhibit the adsorption of VMAs, which, in turn, enhances their stabilizing effects.

14 In addition, adsorption and desorption kinetics could be at the origin of the evolution of

15 material rheological behavior over time. In some cases, the exchange could be rapid, as

16 the desorption of a superplasticizer by sulfate (K. Yamada et al., 2001) or sodium

17 gluconate ( $\mathrm{H}$. Bessaies-Bey et al, 2016). However, as the desorption of an already

18 adsorbed macromolecule requires the simultaneous desorption of the anchor groups,

19 the exchange could also be a very slow process in the order of hours, days or even years

20 (J.A. Kling et al., 1998).

21 To control the outcome of competitive adsorption, several recent studies focused on the

22 modification of the chemical structure and surface affinity of the organic admixtures (W.

23 Fan et al., 2012), (S. Pourchet et al., 2012), (F. Dalas et al., 2015). Moreover, it should be

24 kept in mind that competitive adsorption is only observed at high surface saturation (i.e.

25 high surface coverage) where there are not enough sites for adsorbing species. The

26 extent of this regime can therefore be controlled by tuning the polymer dosage,

27 adsorbed conformation or adsorption energy.

\subsection{Admixtures, hydration and structuration}


1 Admixtures do not only modify the colloidal interactions at the origin of the yield stress

2 and elasticity of the material. They may also affect the hydration reaction in the

3 induction period and modify therefore the structuration at rest of the fresh suspension

4 and the associated evolutions in yield stress and elastic modulus (see Section 2.2.). As

5 for most of the admixtures that have the ability to adsorb on cement particles, PCEs, for

6 example, affect hydration by delaying the setting and disturbing the chemical balance

7 between the cement phases (Cheung et al., 2011), (D. Marchon and R.J. Flatt, 2015), (L.

8 Berodier et al., 2018), (Jansen et al., 2012). Among them, $C_{3} S$ and $C_{3} A$ are the most

9 reactive and therefore the most relevant phases regarding the influence of their

10 hydration on the evolution of the rheological properties. In the first minutes after

11 contact with water, the principal hydration mechanisms are their dissolution and the

12 nucleation and growth of their hydrates. $\mathrm{C}_{3} \mathrm{~A}$ reacts with calcium sulfate and water to

13 form ettringite, and $\mathrm{C}_{3} \mathrm{~S}$ hydration produces portlandite and $\mathrm{C}-\mathrm{S}-\mathrm{H}$, for which we have

14 seen in section 2.2. the major role in the structuration of cement-based materials at

15 rest.

16 Several reasons were mentioned for the delay of hydration caused by PCEs: the

17 complexation with Ca-ion, the perturbation of hydrates formation and the slowing down

18 of $\mathrm{C}_{3} \mathrm{~S}$ dissolution (D. Marchon and R.J. Flatt, 2015). Recent findings firstly highlight the

19 blocking of active dissolution sites by adsorbed molecules as the most realistic

20 explanation for the change of hydration kinetics that leads to a delay of cement setting

21 (D. Marchon et al., 2017). Secondly, a direct quantified correlation was found between

22 the retardation and the molecular structure and dosage of the PCEs (Fig. 7). This led for

23 the first time to the possibility of mastering both rheological properties and side effects

24 of using PCEs with respect to their molecular characteristics and therefore to an

25 optimization of the molecular design (D. Marchon et al, 2019a), (D. Marchon et al, 26 2019b).

27 Although the ions complexation and the inhibition or change of hydrates precipitation

28 seem to have less impact on the decrease of reaction rate of cement, their influence on

29 the location and the way hydrates develop is of great relevance regarding structuration 
1 build-up and microstructure development (P.J.M. Monteiro et al., 2019). In $C_{3} S$ pastes,

2 nano-clusters composed of calcium and polymer complexes were observed in the pore

3 solution (Sowoidnich et al., 2015). This might be linked to the recent findings that PCEs

4 stabilize globular precursors of purely synthesized $\mathrm{C}-\mathrm{S}-\mathrm{H}$ before they convert to the 5 more commonly observed nano-foil like structure (Schönlein and Plank, 2018).

6 Nanoparticles composed of polymer and hydrate phases were also observed in pore

7 solution of cement pastes (Caruso et al. 2017). The presence of such particles in the

8 aqueous solution dispersed by the PCEs could most probably be at the origin of a

9 transition from a heterogeneous to homogeneous nucleation (Valentini et al., 2016),

10 that would affect the bridging effect of $\mathrm{C}-\mathrm{S}-\mathrm{H}$ mentioned earlier as well as the final

11 spatial distribution of the phases in the microstructure (Artioli et al., 2014).

12 Finally, a recent study focusing on the workability loss of cement pastes containing PCEs

13 highlighted two very distinct and successive regimes (S. Mantellato et al., 2019). The

14 first one occurring along the induction period shows a linear evolution of the yield stress

15 that is independent of hydration reactions, illustrating the thixotropic behavior of the

16 system. After a so-called onset, the measured exponential increase in yield stress is

17 directly proportional to the heat rate and to the specific surface area of the system. This

18 correlation points out the dependency of rheological properties on the strong

19 precipitation of hydrates in the acceleration period. With respect to PCEs influence, it

20 was observed that this proportionality changes in presence of the polymer, due to the

21 retardation effect, but does not seem to closely depend on the molecular structure of

22 the PCEs.

23 More generally, admixtures are essential for applications such as concrete 3D printing,

24 where a structural build-up is needed right after deposition of the concrete and

25 followed by a fast stiffening to sustain subsequent layers (L. Reiter et al., 2018). Playing

26 along with admixtures and targeting silicates along with aluminates seem to show great

27 potential for printing concrete (D. Marchon et al., 2018). For example, this can be done

28 by enhancing ettringite precipitation with shotcrete accelerators or blended cements

29 with calcium aluminate cement for a fast surface development and rapid strength gain. 
1 However, the challenge is to choose the perfect mixture proportions between the

2 different systems that are available to avoid an uncontrolled expansion or to not disturb

3 the balance between the main phases of the system. Indeed, provoking ettringite

4 nucleation or having a high aluminate content might strongly influence the sulfate

5 consumption and silicate hydration (Cheung et al., 2011) (L. Berodier et al., 2018). In

6 term of chemistry of hydration, the sulfate depletion is strongly accelerated and if it

7 occurs before the strong precipitation of $\mathrm{C}-\mathrm{S}-\mathrm{H}$, the presence of a very high content of

8 aluminum in the pore solution inhibits the dissolution of the silicate phase and therefore

9 delays the precipitation of $\mathrm{C}-\mathrm{S}-\mathrm{H}$ that is the source of the mechanical strength $(\mathrm{N}$.

10 Tenoutasse, 1968), (E. Pustovgar et al., 2017). This has a detrimental impact on the early

11 as well as on the late mechanical strength. In term of rheology, this causes a very rapid

12 stiffening, called flash set, and subsequent loss of fluidity.

\subsection{Nanoparticles and structuration}

15 It was shown by (P. Juilland et al., 2012) that the mechanical action of mixing of cement 16 paste results in the detachment of $\mathrm{C}-\mathrm{S}-\mathrm{H}$ from the surface of particles leading to the 17 enhancement of the main heat peak of hydration. Recent work by (F. Caruso et al., 18 2017) highlighted the formation of nanoparticles in cement pore solution containing 19 super-plasticizers. They asserted that these nano-size particles are either nano-C-S-H, 20 nano-ettringite, nano-AFm, or intramolecular complexes of polymer and cations. More 21 recently, (A. Vandenberg et al., 2018) showed that an appropriate combination of 22 mixing energy and super-plasticizer dosage promotes the scratching of nanoparticles 23 from the surface of cement particles and their stabilization in the suspending fluid and 24 hence generates additional nucleation surfaces. These generated nanoparticles enhance 25 the thixotropic behavior of cement-based materials and promote structuration. 
1 In addition, it is possible to further tailor nucleation and structuration by introducing

2 nanoparticles as additives. Highly purified attapulgite nanoclays have been

3 demonstrated to be effective in enhancing thixotropy at relatively low additions (S.

4 Kawashima et al., 2013), (Z. Quanji et al., 2014), (Y. Qian and S. Kawashima, 2016). Their

5 effect has been tied to the colloidal strain regime, which supports that the clays are

6 enhancing electrostatic interactions between cement particles to enhance yield stress

7 recovery immediately after shear (S. Ma et al., 2018). Due to their high specific surface

8 area, nanoparticles can introduce additional CSH nucleation sites to accelerate

9 hydration, termed seeding effect, (F. Sanchez and K. Sobolev, 2010), which can in turn

10 enhance structuration in the fresh state. One challenge of incorporating any

11 nanoparticle type in cement systems is dispersion, where dispersion degree will

12 significantly impact the working mechanism. Therefore the seeding effect is sensitive to

13 processing parameters, i.e. dispersion technique and mixing. This is the underlying

14 reason why, in contrast to flow onset results where increase in yield stress is

15 consistently observed, the effect of nanoclay on SAOS results is much more dependent

16 on processing and pre-shear conditions (S. Kawashima et al., 2013), (T. Conte and M.

17 Chaouche, 2016), (S. Ma et al., 2018).

\section{Yield stress and elasticity in fresh cement-based materials: modeling}

\subsection{Yield stress modeling and YODEL}

21 The YODEL is a first principle analysis of yield stress of colloidal particles suspension, 22 which was shown to successfully predict the main parametric dependencies of cement 23 suspensions yield stress (R.J. Flatt and P. Bowen, 2006), (R.J. Flatt and P. Bowen, 2007)

24 (A. Perrot et al., 2012), (J. Hot et al., 2014). This model writes:

$$
\tau_{P} \cong m \frac{A_{0} a^{*} \varphi_{c}^{2}\left(\varphi_{c}-\varphi_{p e r c}\right)}{d^{2} H^{2} \varphi_{c m}\left(\varphi_{c m}-\varphi_{c}\right)}
$$

25 Where we have 5 material parameters:

$26 m$ is a pre-factor, which depends on the cement particle size distribution; It can be computed from a measurement of the particle size distribution. 
$1 A_{0}$ is the non-retarded Hamaker constant taken as $1.6 \times 10^{-20} \mathrm{~J}$;

$2 a^{*}$ is typical size of the surface defects of cement grains and is of the order of several

3 hundreds of $n m$ (A. Perrot et al., 2012);

$4 d$ is the cement particle average diameter $d_{50}$ and is of the order of $10 \mu \mathrm{m}$

$5 \varphi_{c m}$ is the maximum packing fraction of cement grains.

6 And 3 mixture variables:

$7 \varphi_{c}$ is the solid volume fraction of the cement particles in cement paste

$8 \varphi_{\text {perc }}$ is the percolation volume fraction; It results from the competition between

9 Brownian motion and colloidal attractive forces between cement particles, which, in 10 turn, depend on the admixture dosage (A. Perrot et al., 2012).

$11 H$ is the average surface-to-surface separating distance between flocculated cement 12 particles; It depends on the admixture dosage.

\subsection{Flow and processing modeling}

15 The previous sections have introduced the experiments allowing for the measurement 16 of yield stress and the model allowing for the prediction of yield stress as a function of 17 components properties and dosage. The present section refers to the mathematical 18 models that link yield stress to the macroscopic flow behaviour, by introducing its value 19 into macroscopic flow equations. As mentioned in the introduction, the common 20 approach to model fresh cementitious materials is based on non-Newtonian fluid 21 mechanics. Here, the macroscopic flow behaviour is expressed by constitutive 22 equations, which give relation between the shear stresses and velocity gradients (J.D. 23 Anderson, 1992). The commonly accepted constitutive relations for fresh cement-based 24 materials are Bingham and Herschel-Bulkley (C.F. Ferraris, 1999). The Bingham model 25 (Equation 2) captures a linear viscous behaviour if the applied stress is higher than the 26 yield value (E.C. Bingham, 1922), while the Herschel-Bulkley approach (W.H. Herschel 27 and R. Bulkley, 1926) assumes that the material behaves non-linearly if the stresses are 28 above yield stress value (Equation 3):

$$
\tau=\tau_{0}+\eta_{p l} \cdot \dot{\gamma}
$$




$$
\tau=\tau_{0}+k \cdot \dot{\gamma}^{n}
$$

1 where $\tau$ is shear stress, $\tau_{0}$ is yield stress of the material, $\eta_{p l}$ is plastic viscosity of the 2 material, $\dot{\gamma}$ is the shear rate, $\mathrm{k}$ is consistency index and $\mathrm{n}$ is power law index. The 3 constitutive relations are integrated into the conservation equations, which govern the 4 fluid flow.

5 The abovementioned mathematical models found practical applications in numerical

6 concrete flow simulations (N. Roussel and A. Gram, 2014), (A. Gram and J. Silfwerbrand, 7 2011), (L.N. Thrane, 2012). Due to the computational limits, a complete flow simulation 8 that account for all concrete phases is still not possible; therefore, the above authors 9 used different approximate models that aim to describe the macroscopic flow behavior 10 (rather than its microstructure). Recently, in (N. Roussel et al., 2016) the researchers 11 studied numerically the effect of yield stress on the final shape in two concrete filling 12 ability tests: slump and channel flow. They compared the numerical predictions of these 13 benchmark flows obtained by several research teams, who used various numerical tools. 14 The comparison demonstrated that, if segregation does not occur, the compared 15 numerical simulations are able to accurately predict the benchmark flows, and concrete 16 filling ability. The numerical results were in agreement with the analytical solutions 17 proposed in (N. Roussel, 2007), (N. Roussel and P. Coussot, 2005), which claim that final 18 shape and flow distance of a yield-stress material are determined by its density and the 19 yield stress value, only.

20 The findings of this numerical benchmark study are of particular interest for the 21 practical application of numerical simulations to predict industrial processes. The 22 purpose of a numerical prediction is not to perfectly depict the process itself, but to 23 predict its result (for instance, in case of concreting on site, one aims to predict if the 24 formwork will be properly filled or not). Consequently, such a simulation focuses on the 25 flow at stoppage, where the shear rates are rather low, and the contribution of the 26 second part of the equations 2 and 3 is negligible. It is therefore not crucial if the 27 material behaves linearly or non-linearly at the higher shear rates - at the low shear 28 rates at stoppage the yield stress is the dominating factor, that finally determines the 
1 flow length or the filling degree of a mold. This was recently demonstrated in (K. Vasilic

2 et al. 2016), through large scale-laboratory castings and corresponding numerical

3 simulations: for non-blocking cases, yield stress determines the final shape of the

4 material and the proper filling of the form in practical applications.

\subsection{Stability modeling of printed cement-based materials}

7 The successful deposition of consecutive layers in a 3D printing process lead to an

8 increment of gravity-induced stresses as an object is created. As the resistance of the

9 printing material is initially low (i.e. in the order of the loads acting on the material) and

10 no confining formwork is present, this increment in stress may lead to structural failure

11 during the printing process, by either elastic buckling (illustrated in Figure 9) or plastic

12 collapse. In this phase, when the fresh concrete is generally at rest, the elastic-plastic

13 behavior of the early age material, and its structuration kinetics, are critical to control

14 such failure (N. Roussel et al., 2012), (L. Reiter et al., 2018).

15 In a recent paper, the rheological requirements to prevent both material yielding as well

16 as self-buckling have been outlined (N. Roussel, 2018). It was shown that, for a typical

17 wall of $2.5 \mathrm{~m}$ height, the gravity-induced stresses gradually increase with the rising

18 height of the object, and reach a value of around $30 \mathrm{kPa}$ in the critical bottom layer at

19 the end of printing.

20 Consequently, in addition to an initial material yield stress required to control the layer

21 shape immediately after extrusion (typically in the order of a couple tens to thousands

22 of $\mathrm{Pa}$ ), a structuration rate $A_{\text {thix }}(\mathrm{N}$. Roussel, 2006) is imposed to prevent plastic collapse

23 when consecutive layers are placed. The minimum rate is dependent on the rising speed

24 of the object, and may follow both a linear and non-linear trend (A. Perrot et al., 2015),

25 (T. Wangler et al., 2016). Likewise, the initial elasticity and its evolution rate required to

26 avoid elastic buckling, a common failure mode for the dominantly vertical slender

27 shapes created by 3D printing, are imposed by the rising speed of the object, as well as

28 its geometrical features. The rising speed, in turn, follows from the printing velocity, 
1 contour length, and layer dimensions. Thus, the rheological requirements of 3D printed

2 concrete are fully process dependent.

3 The occurrence of failure by elastic buckling in a 3D concrete printing process was for

4 the first time shown in a recent paper (R. Wolfs et al., 2018). To assess the process

5 dependent structural failure of an object during 3D printing, the authors proposed a

6 numerical approach based on Finite Element Method (FEM) modelling. This approach

7 includes the development of both the geometry and the material properties throughout

8 a printing process, and thus, may predict if, and how, an object may collapse. The

9 numerical simulations were validated by means of printing experiments. With a similar

10 aim, a mechanistic model has recently been presented, to explore the influence of

11 various process parameters on the mechanical performance of 3D printed wall

12 structures (A.S.J. Suiker, 2018).

13 The recent developments in the field of $3 \mathrm{D}$ concrete printing highlight the need to

14 assess both yield stress and elasticity beyond the range commonly considered for fresh

15 concrete. Whereas the rheological properties of concrete are often studied in the range

16 of several $\mathrm{Pa}$, they may reach the order of several tens to thousands of $\mathrm{kPa}$ in a 3D

17 printing process. In this range, standard rheometers may not be adequate, which has led

18 to the adoption of mechanical tests to characterize the early age properties. The results

19 of such experiments additionally demonstrate a decrease of the critical strain with time

20 at rest, which indicates a transition from plastic towards brittle failure as the material

21 ages (L.K. Mettler et al., 2016), (R. Wolfs et al. 2018). Consequently, where fresh

22 concrete is often considered as a non-Newtonian fluid with a Von Mises yield criterion,

23 at later ages, a solid mechanics approach based on a Mohr-Coulomb or Drucker-Prager

24 criterion may be more suitable to assess plastic collapse in 3D printing processes. 


\section{CONCLUSION}

3 This paper gathered the accepted knowledge along with the most recent advances on

4 yield stress and elasticity of fresh cement-based materials. In the first part, the physical 5 and chemical origins of these macroscopic properties and their evolutions were 6 described. The second part of the paper focused on the way these properties can be 7 measured while the third part dealt with the way they can be controlled and tuned.

8 Finally, the most recent models for both properties prediction from mix design and

9 processing modeling from rheological measurements were described.

10 The clear distinction in the research community between non-Newtonian fluid 11 mechanics and fresh cement-based materials on one side and solid mechanics and 12 hardened ones on the other side lasted for decades. However, it can be concluded from 13 the above research trends that, nowadays, the boundary between these two fields is 14 becoming narrow.

15 Because of the need to measure, understand and tune rheological properties of 16 printable materials, the rheology and processing community is now exploring 17 consistency and strength regimes that used to be considered as typical of a hardening 18 material. In parallel, the hydration community keeps on improving its understanding of 19 the setting process. Although being an extremely short phenomena relative to the life 20 period of a concrete element, yield stress, elasticity, thixotropy, structuration and 21 setting seem to be progressively described in literature as a continuous transition with 22 the same physico-chemical origin.

\section{ACKNOWLEDGEMENT}

25 This work has been carried out by Nicolas Roussel and Hela Bessaies-Bey within the 26 frame of the project DiXite. Initiated in 2018, DiXite is part of I-SITE FUTURE, a French 27 initiative to answer the challenges of sustainable cities. Shiho Kawashima would like to 28 acknowledge the National Science Foundation (NSF \#1653419) for financial support 29 while financial support for Delphine Marchon was provided by the Swiss National 30 Science Foundation (Early Postdoc Mobility grant no. P2EZP2-172177). 


\section{REFERENCES}

2 A. Gram, J. Silfwerbrand, "Numerical simulation of fresh SCC flow: applications", Mater. 3 Struct., 44 (2011) pp. 805-813.

4 A. Nonat, J.C. Mutin, X. Lecoq, S.P. Jiang, Physico-chemical parameters determining 5 hydration and particle interactions during the setting of silicate cements, Solid State 6 Ionics 101-103 (1997) 923-930.

7 A. Papo, A., B. Caufin, A study of the hydration process of cement pastes by means of

8 oscillatory rheological techniques, Cement and concrete research, 21(6) (1991) 111191117.

10 A. Perrot, T. Lecompte, H. Khelifi, C. Brumaud, J. Hot, N. Roussel, Yield stress and 11 bleeding of fresh cement pastes, Cement and Concrete Research, 42(7) (2012) pp. 93712944.

13 A. Perrot, A. Pierre, S. Vitaloni, V. Picandet, Prediction of lateral form pressure exerted 14 by concrete at low casting rates, Materials and Structures, 48(7) (2015) pp. 2315-2322.

15 A. Perrot, D. Rangeard, A. Pierre, Structural built-up of cement-based materials used for 16 3D printing extrusion techniques, Materials and Structures, 49(4) (2016) pp. 1213-1220.

17 A. Vandenberg, H. Bessaies-Bey, K. Wille, N. Roussel, Enhancing Printable Concrete 18 Thixotropy by High Shear Mixing. In: Wangler T., Flatt R. (eds) First RILEM International 19 Conference on Concrete and Digital Fabrication - Digital Concrete 2018. DC 2018. RILEM 20 Bookseries, vol 19. Springer

21 A.M. Mostafa, A. Yahia, New approach to assess build-up of cement-based suspensions, 22 Cement and Concrete Research, 85 (2016) 174-182.

23 A.M. Mostafa, A. Yahia, Physico-chemical kinetics of structural build-up of neat cement24 based suspensions, Cement and Concrete Research, 97 (2017) 11-27.

25 A.S.J. Suiker, Mechanical performance of wall structures in 3D printing processes: 26 Theory, design tools and experiments, Int. J. Mech. Sc. 137 (2018) 145-170.

27 A.W. Saak, H.M. Jennings, S. Shah, A generalized approach for the determination of yield 28 stress by slump and slump flow, Cem. Concr. Res., 34 (2004) pp. 363-371.

29 B. Feneuil, O. Pitois, N. Roussel. Effect of surfactants on the yield stress of cement paste, 
Cem. Concr. Res. 100, 2017, 32-39.

2 C. Brumaud, Origines microscopiques des conséquences rhéologiques de l'ajout d'éthers

3 de cellulose dans une suspension cimentaire, (PhD Thesis) Université Paris-Est, 2011, (In 4 French).

5 C. Brumaud, R. Baumann, M. Schmitz, M. Radler, N. Roussel, Cellulose ethers and yield 6 stress of cement pastes, Cem. Concr. Res. 55 (2014) 14-21.

7 C. F. Ferraris, Measurement of the rheological properties of high performance concrete;

8 State of the art report, J. Res. Natl. Inst. Stand. Technol. 104 (1999) 461-478.

9 D. Feys, R. Cepuritis, S. Jacobsen, K. Lesage, E. Secrieru, A. Yahia, Measuring Rheological 10 Properties of Cement Pastes: Most common Techniques, Procedures and Challenges, 11 RILEM technical letters, pp. 129-135, 2017.

12 D. Jansen, J. Neubauer, F. Goetz-Neunhoeffer, R. Haerzschel, W.-D. Hergeth, Change in 13 reaction kinetics of a Portland cement caused by a superplasticizer - Calculation of heat 14 flow curves from XRD data, Cement and Concrete Research. 42 (2012) 327-332.

15 D. Lowke, Thixotropy of SCC-A model describing the effect of particle packing and 16 superplasticizer adsorption on thixotropic structural build-up of the mortar phase based 17 on interparticle interactions, Cement and Concrete Research, 104 (2018) 94-104.

18 D. Marchon, U. Sulser, A. Eberhardt, R.J. Flatt, Molecular design of comb-shaped 19 polycarboxylate dispersants for environmentally friendly concrete, Soft Matter. 9 (2013) $20 \quad 10719-10728$.

21 D. Marchon, P. Juilland, E. Gallucci, L. Frunz, R.J. Flatt, Molecular and submolecular scale 22 effects of comb-copolymers on tri-calcium silicate reactivity: Toward molecular design, 23 Journal of the American Ceramic Society. 100 (2017) 817-841. doi:10.1111/jace.14695.

24 D. Marchon, R.J. Flatt, 12 - Impact of chemical admixtures on cement hydration, in: P.-C. 25 Aïtcin, R.J. Flatt (Eds.), Science and Technology of Concrete Admixtures, Woodhead 26 Publishing, 2016: pp. 279-304.

27 D. Marchon, S. Kawashima, H. Bessaies-Bey, S. Mantellato, S. Ng, Hydration and 28 rheology control of concrete for digital fabrication: Potential admixtures and cement 29 chemistry, Cement and Concrete Research. 112 (2018) 96-110. 
1 E. Berodier, L.R. Gibson, E. Burns, L. Roberts, J. Cheung, Robust production of 2 sustainable concrete through the use of admixtures and in-transit concrete 3 management systems, Cement and Concrete Composites (2018), in press.

4 D. Marchon, F. Boscaro, R.J. Flatt, First steps to the molecular structure optimization of 5 polycarboxylate ether superplasticizers: Mastering fluidity and retardation, Cement and 6 Concrete Research. 115 (2019) 116-123.

7 E. C. Bingham, Fluidity and plasticity, first ed., Mcgraw-Hill Book Company, Inc., New 8 York, 1922.

9 E. Meyer, K. Rosenberg, J. Israelachvili, Recent progress in understanding hydrophobic 10 interactions, PNAS 103 (43) (2006) 15739-15746.

11 E. Pustovgar, R.K. Mishra, M. Palacios, J.-B. d'Espinose de Lacaillerie, T. Matschei, A.S.

12 Andreev, H. Heinz, R. Verel, R.J. Flatt, Influence of aluminates on the hydration kinetics

13 of tricalcium silicate, Cement and Concrete Research. 100 (2017) 245-262.

14 F. Caruso, S. Mantellato, M. Palacios, R.J. Flatt, ICP-OES method for the characterization 15 of cement pore solutions and their modification by polycarboxylate-based 16 superplasticizers, Cem. Concr. Res. 91 (2017) 52-60.

17 F. Dalas, A. Nonat, S. Pourchet, M. Mosquet, D. Rinaldi, S. Sabio, Tailoring the anionic 18 function and the side chains of comb-like superplasticizers to improve their adsorption, 19 Cem. Concr. Res. 67 (2015) 21-30.

20 F. Mahaut, S. Mokeddem, X. Chateau, N. Roussel, G. Ovarlez, Effect of coarse particle 21 volume fraction on the yield stress and thixotropy of cementitious materials, Cem. 22 Concr. Res. 38(11) (2008b) pp. 1276-1285.

23 F. Mahaut, X. Chateau, P. Coussot, G. Ovarlez, Yield stress and elastic modulus of 24 suspensions of non-colloidal particles in yield stress fluids, J. Rheol., 52 (2008a) pp. 28725313.

26 F. Sanchez, K. Sobolev, Nanotechnology in concrete-a review, Construction and building 27 materials, 24(11) (2010) 2060-2071.

28 G. Artioli, L. Valentini, M. Voltolini, M.C. Dalconi, G. Ferrari, V. Russo, Direct Imaging of 29 Nucleation Mechanisms by Synchrotron Diffraction Micro-Tomography: 
1 Superplasticizer-Induced Change of C-S-H Nucleation in Cement, Crystal Growth \&

2 Design. 15 (2015) 20-23.

3 G. Gelardi, R.J. Flatt, 11 - Working mechanisms of water reducers and superplasticizers,

4 in: P.-C. Aïtcin, R.J. Flatt (Eds.), Science and Technology of Concrete Admixtures,

5 Woodhead Publishing, 2016: pp. 257-278.

6 G. Gelardi, S. Mantellato, D. Marchon, M. Palacios, A.B. Eberhardt, R.J. Flatt, 9 -

7 Chemistry of chemical admixtures, in: P.-C. Aïtcin, R.J. Flatt (Eds.), Science and

8 Technology of Concrete Admixtures, Woodhead Publishing, 2016: pp. 149-218.

9 H. Bessaies-Bey, R. Baumann, M. Schmitz, M. Radler, N. Roussel, Effect of 10 polyacrylamide on rheology of fresh cement pastes, Cem. Concr. Res. 76 (2015) 98-106.

11 H. Bessaies-Bey, R. Baumann, M. Schmitz, M. Radler, N. Roussel, Organic admixtures and 12 cement particles: Competitive adsorption and its macroscopic rheological 13 consequences, Cem. Concr. Res. 80 (2016) 1-9.

14 H. Bessaies-Bey, M. Palacios, E. Pustovgar, M. Hanafi, R. Baumann, R.J. Flatt, N. Roussel, 15 Non-adsorbing polymers and yield stress of cement paste: Effect of depletion forces, 16 Cement and Concrete Research. 111 (2018) 209-217.

17 J. Assaad, K.H. Khayat, H. Mesbah, Assessment of thixotropy of flowable and self18 consolidating concrete, Materials Journal, 100(2) (2003a) 99-107.

19 J. Assaad, K.H. Khayat, H. Mesbah, Variation of formwork pressure with thixotropy of 20 self-consolidating concrete, Materials Journal, 100(1) (2003b) 29-37.

21 J. Cheung, A. Jeknavorian, L. Roberts, D. Silva, Impact of admixtures on the hydration 22 kinetics of Portland cement, Cement and Concrete Research. 41 (2011) 1289-1309.

23 J. D. Anderson Jr., Governing Equations of Fluid Dynamics, in: J. Wendt, (Ed.), 24 Computational Fluid Dynamics, Springer Berlin Heidelberg, 1992, 15-51.

25 J. Hot, H. Bessaies-Bey, C. Brumaud, M. Duc, C. Castella, C., N. Roussel, Adsorbing 26 polymers and viscosity of cement pastes, Cement and Concrete Research, 63 (2014) pp. $27 \quad 12-19$.

28 J. Israelachvili, R. Pashley, Measurement of the hydrophobic interaction between two 29 hydrophobic surfaces in aqueous electrolyte solutions, J. Colloid Interface Sci. 98 (2) 
1 (1984).

2 J. Murata, Flow and deformation of fresh concrete, Materials and Structures 98 (1984)

3 pp. 117-129.

4 J. Plank, A. Brandl, N.R. Lummer, Effect of different anchor groups on adsorption

5 behavior and effectiveness of poly(N,N-dimethylacrylamide-co-Ca 2-acrylamido-2-

6 methylpropanesulfonate) as cement fluid loss additive in presence of acetone-

7 formaldehyde-sulfite dispersant, J. Appl. Polym. Sci. 106 (2007) 3889-3894.

8 J. Plank, C. Winter, Competitive adsorption between superplasticizer and retarder

9 molecules on mineral binder surface, Cem. Concr. Res. 38 (2008) 599-605.

10 J. Plank, N.R. Lummer, F. Dugonjić-Bilić, Competitive adsorption between an AMPS ${ }^{\circledR}$

11 based fluid loss polymer and Welan gum biopolymer in oil well cement, J. Appl. Polym.

12 Sci. 116 (2010) 2913-2919.

13 J. Yammine, M. Chaouche, M. Guerinet, M. Moranville, N. Roussel From ordinary

14 rheology concrete to self compacting concrete : a transition between frictional and

15 hydrodynamic interactions, Cement and Concrete Research, 38 (2008) pp. 890-896.

16 J.A. Kling, H.J. Ploehn, Desorption of Adsorbed Poly(ethylene Oxide) from Colloidal

17 Polystyrene Particles, J. Colloid Interface Sci. 198 (1998) 241-248.

18 J.E. Wallevik, Rheological properties of cement paste: thixotropic behavior and

19 structural breakdown, Cement and Concrete Research, 39 (2009) 14-29.

20 K. Vasilic, W. Schmidt, H.C. Kühne, F. Haamkens, V. Mechtcherine, N. Roussel, Flow of

21 fresh concrete through reinforced elements: experimental validation of the porous

22 analogy numerical method, Cem. Concr. Res. 88 (2016), 1-6.

23 K. Yamada, S. Ogawa, S. Hanehara, Controlling of the adsorption and dispersing force of

24 polycarboxylate-type superplasticizer by sulfate ion concentration in aqueous phase,

25 Cem. Concr. Res. 31 (2001) 375-383.

26 L. Josserand, O. Coussy, F. de Larrard, Bleeding of concrete as an ageing consolidation

27 process, Cement and Concrete Research, 36(9) (2006) pp. 1603-1608.

28 L. Nachbaur, J.C. Mutin, A. Nonat, L. Choplin, Dynamic mode rheology of cement and

29 tricalcium silicate pastes from mixing to setting, Cem. Concr. Res. 31 (2001) 183-192. 
1 L. Reiter, T. Wangler, N. Roussel, R.J. Flatt, The role of early age structural build-up in

2 digital fabrication with concrete, Cem. Concr. Res. 112 (2018) 86-95.

3 L. Valentini, M. Favero, M.C. Dalconi, V. Russo, G. Ferrari, G. Artioli, Kinetic Model of

4 Calcium-Silicate Hydrate Nucleation and Growth in the Presence of PCE

5 Superplasticizers, Crystal Growth \& Design. $16 \quad$ (2016) 646-654.

6 doi:10.1021/acs.cgd.5b01127.

7 L.K. Mettler, F.K. Wittel, R.J. Flatt, H.J. Herrmann, Evolution of strength and failure of

8 SCC during early hydration, Cem. Concr. Res. 89 (2016) pp. 288-296.

9 L.N. Thrane, Modelling the flow of self-compacting concrete, in: N. Roussel (Ed.),

10 Understanding Rheology of concrete, Woodhead Publ Ltd, Cambridge 2012, 259-285.

11 M. Schönlein, J. Plank, A TEM study on the very early crystallization of C-S-H in the

12 presence of polycarboxylate superplasticizers: Transformation from initial C-S-H globules

13 to nanofoils, Cement and Concrete Research. 106 (2018) 33-39.

14 M.A. Schultz, L. Struble, Use of oscillatory shear to study flow behavior of fresh cement

15 paste, Cement and Concrete Research, 23 (1993) 273-282.

16 N. Massoussi, E. Keita, N. Roussel, The heterogeneous nature of bleeding in cement 17 pastes, Cem. Concr. Res., 95 (2017) pp. 108-116.

18 N. Pashias, D.V. Boger, J. Summers, D.J. Glenister, A fifty cent rheometer for yield stress 19 measurements, J. of Rheol., 40(6) (1996) pp. 1179-1189.

20 N. Roussel, P. Coussot, Fifty-cent rheometer for yield stress measurements: From slump 21 to spreading flow, J. Rheol. 49 (2005) 705-718.

22 N. Roussel, C. Stefani, R. Leroy, From mini cone test to Abrams cone test : measurement 23 of cement based materials yield stress using slump tests, Cement and Concrete 24 Research, 35(5) (2005) 817-822.

25 N. Roussel, Correlation between yield stress and slump : Comparison between numerical

26 simulations and concrete rheometers results, Materials and Structures, 39(4) (2006) pp.

$27 \quad 501-509$.

28 N. Roussel, A thixotropy model for fresh fluid concretes: theory, validation and 29 applications, Cem. Concr. Res. 36 (10) (2006) 1797-1806. 
1 N. Roussel, Rheology of fresh concrete: from measurements to predictions of casting

2 processes, Materials and Structures, Volume: 40 Issue: 10 Pages: 1001-1012, (2007a).

3 N. Roussel, The LCPC BOX: a cheap and simple technique for yield stress measurements

4 of SCC, Mater. Struct. 40 (2007b) pp. 889-896.

5 N. Roussel, A. Lemaitre, A., R.J. Flatt, P. Coussot, Steady state flow of cement

6 suspensions: A micromechanical state of the art, Cement and Concrete Research,

7 Volume: 40 Issue: 1 Pages: 77-84, 2010.

8 N. Roussel, G. Ovarlez, S. Garrault, C. Brumaud, The origins of thixotropy of fresh cement

9 pastes, Cem. Concr. Res. 42 (1) (2012) 148-157.

10 N. Roussel, A. Gram (Eds.), Simulation of Fresh Concrete Flow, State-of-the Art Report of 11 the RILEM Technical Committee 222-SCF, Springer, Netherlands, 2014.

12 N. Roussel, A. Gram, M. Cremonesi, L. Ferrara, K. Krenzer, V. Mechtcherine, S. Shyshko,

13 J. Skocec, J. Spangenberg, O. Svec, L. N. Thrane and K. Vasilic, Numerical simulations of

14 concrete flow: A benchmark comparison, Cem. Concr. Res. 79, (2016), 265-271.

15 N. Roussel, Rheological requirements for printable concretes, Cem. Concr. Res. 112 16 (2018) 76-85.

17 N. Tenoutasse, The Hydratation Mechanism of C3A and C3S in the Presence of Calcium 18 Chloride and Calcium Sulphate, CRIC, 1969.

19 P. Billberg, (2003), "Form pressure generated by self-compacting concrete", 20 Proceedings of the 3rd international RILEM Symposium on Self-Compacting Concrete,

21 RILEM PRO33 Reykjavik, Iceland, pp. 271-280.

22 P. Juilland, A. Kumar, E. Gallucci, R.J. Flatt, K.L Scrivener: Effect of mixing on the early 23 hydration of alite and OPC systems. Cem.Concr.Res. 42, 1175-1188 (2012).

24 P.F.G. Banfill, R.E. Carter, P.J. Weaver, Simultaneous rheological and kinetic 25 measurements on cement pastes, Cement and Concrete Research, 21(6) (1991) 1148261154.

27 P.J.M. Monteiro, G. Geng, D. Marchon, J. Li, P. Alapati, K.E. Kurtis, M.J.A. Qomi, 28 Advances in characterizing and understanding the microstructure of cementitious 29 materials, same issue (2019). 
1 Q. Yuan, D. Zhou, K.H. Khayat, D. Feys, C. Shi, On the measurement of evolution of 2 structural build-up of cement paste with time by static yield stress test vs. small 3 amplitude oscillatory shear test, Cement and Concrete Research, 99 (2017) 183-189.

4 R.J. Flatt, Y.F. Houst, P. Bowen, H. Hofmann, J. Widmer, U. Sulser, U. Maeder, T.A. Bürge, 5 Interaction of superplasticizers with model powders in a highly alkaline medium, in: Proc

6 5th CANMETACI Intern Conf Superplast. Chem. Admix. Concr., American Concrete

7 Institute, Farmington Hills, Mi, USA, 1997: pp. 743-762.

8 R.J. Flatt, P. Bowen, Yodel: A Yield Stress Model for Suspensions, Journal of American

9 Ceramic Society, Vol. 89 (4), pp. 1244-1256, (2006).

10 R.J. Flatt, P. Bowen, Yield Stress of Multimodal Powder Suspensions : An Extension of 11 the YODEL (Yield Stress mODEL), Journal of American Ceramic Society, Vol. 90(4), pp. 12 1038-1044, (2007).

13 R.J. Flatt, I. Schober, E. Raphael, C. Plassard, E. Lesniewska, Conformation of Adsorbed 14 Comb Copolymer Dispersants, Langmuir. 25 (2009) 845-855.

15 R.J. Flatt, J. Zimmermann, C. Hampel, C. Kurz, I. Schober, L. Frunz, C. Plassard, E. 16 Lesniewska, The Role of Adsorption Energy in the Sulfate-Polycarboxylate Competition, 17 Spec. Publ. 262 (2009) 153-164.

18 R.J. Flatt, I. Schober, 7 - Superplasticizers and the rheology of concrete, in: N. Roussel 19 (Ed.), Understanding the Rheology of Concrete, Woodhead Publishing, 2012: pp. 14420208.

21 R.J.M. Wolfs, F.P. Bos, T.A.M. Salet, Correlation between destructive compression tests 22 and non-destructive ultrasonic measurements on early age 3D printed concrete. Constr. 23 Build. Mater. 181 (106) (2018) 447-54.

24 R.J.M. Wolfs, F.P. Bos, T.A.M. Salet, Early age mechanical behavior of 3D printed 25 concrete: numerical modeling and experimental testing, Cem. Concr. Res. 106 (2018) 26 103-116.

27 S. Asakura, F. Oosawa, On interaction between two bodies immersed in a solution of 28 macromolecules, J. Chem. Phys. 22 (1954) 1255.

29 S. Asakura, F. Oosawa, Interaction between particles suspended in solutions of 
1 macromolecules, J. Polym. Sci. 33 (1958) 183.

2 S. Clayton, T.G. Grice, D.V. Boger, Analysis of the slump test for on-site yield stress

3 measurement of mineral suspensions, Int. J. Miner. Process., 70 (2003) pp. 53-21.

4 S. Kawashima, M. Chaouche, D.J. Corr, S.P. Shah, Rate of thixotropic rebuilding of

5 cement pastes modified with highly purified attapulgite clays, Cement and Concrete

6 Research, 53 (2013) 112-118.

7 S. Ma, Y. Qian, S. Kawashima, Experimental and modeling study on the non-linear

8 structural build-up of fresh cement pastes incorporating viscosity modifying admixtures,

9 Cement and Concrete Research, 108 (2018) 1-9.

10 S. Mantellato, M. Palacios, R.J. Flatt, Relating early hydration, specific surface and flow 11 loss of cement pastes, Mater Struct. 52 (2019) 5.

12 S. Pourchet, S. Liautaud, D. Rinaldi, I. Pochard, Effect of the repartition of the PEG side

13 chains on the adsorption and dispersion behaviors of PCP in presence of sulfate, Cem.

14 Concr. Res. 42 (2012) 431-439.

15 S.P. Jiang, J.C. Mutin, A. Nonat, Studies on mechanisms and physico-chemical 16 parameters at the origin of cement setting. 1. The fundamental processes involved 17 during the cement setting, Cement Concrete Research, 25(4) (1995) 779-789.

18 T. Conte, M. Chaouche, Rheological behavior of cement pastes under Large Amplitude

19 Oscillatory Shear, Cement and Concrete Research, 89 (2016) 332-344.

20 T. Lecompte, A. Perrot, Non-linear modeling of yield stress increase due to SCC 21 structural build-up at rest, Cement and Concrete Research, 92 (2017) 92-97.

22 T. Sowoidnich, T. Rachowski, C. Rößler, A. Völkel, H.-M. Ludwig, Calcium complexation 23 and cluster formation as principal modes of action of polymers used as superplasticizer 24 in cement systems, Cement and Concrete Research. 73 (2015) 42-50.

25 T. Wangler, E. Lloret, L. Reiter, N. Hack, F. Gramazio, M. Kohler, M. Bernhard, B. 26 Dillenburger, J. Buchli, N. Roussel, R. Flatt, Digital concrete: opportunities and 27 challenges, RILEM Tech. Lett. 1 (2016) 67-75.

28 T.F. Tadros, Dispersion of Powders in Liquids and Stabilization of Suspensions, John 29 Wiley \& Sons, 2012. 
1 T.L.H. Nguyen, N. Roussel, P. Coussot Correlation between L-box test and rheological

2 parameters of an homogeneous yield stress fluid, Cement and Concrete Research, 3 36(10) (2006) pp. 1789-1796

4 V. Picandet, D. Rangeard, A. Perrot, T. Lecompte, Permeability measurement of fresh

5 cement paste, Cement and Concrete Research, Volume 41, Issue 3, Pages 330-338,

6 March 2011.

7 W. Fan, F. Stoffelbach, J. Rieger, L. Regnaud, A. Vichot, B. Bresson, N. Lequeux, A new

8 class of organosilane-modified polycarboxylate superplasticizers with low sulfate

9 sensitivity, Cem. Concr. Res. 42 (2012) 166-172. doi:10.1016/j.cemconres.2011.09.006.

10 W.H. Herschel and R. Bulkley, Konsistenzmessungen von Gummi-Benzollösungen,

11 Kolloid Z. (39), 1926, 291-300.

12 W.G. Lei, L.J. Struble, Microstructure and flow behavior of fresh cement paste, Journal of

13 the American Ceramics Society, 80 (8) (1997) 2021-2028.

14 W.R. Schowalter, G. Christensen, Toward a rationalization of the slump test for fresh

15 concrete: comparisons of calculations and experiments, J. of Rheol., 42(4) (1998) pp. $16 \quad 865-870$.

17 Y. Qian, S. Kawashima, Use of creep recovery protocol to measure static yield stress and

18 structural rebuilding of fresh cement pastes, Cement and Concrete Research, 90 (2016)

$19 \quad 73-79$.

20 Z. Quanji, Z., G.R. Lomboy, K. Wang, Influence of nano-sized highly purified magnesium

21 alumino silicate clay on thixotropic behavior of fresh cement pastes, Construction and 22 Building Materials, 69 (2014) 295-300.

23 Z. Sun, T. Voigt, T., S.P. Shah, Rheometric and ultrasonic investigations of viscoelastic 24 properties of fresh Portland cement pastes, Cement and Concrete Research, 36(2) 25 (2006) 278-287.

26

27

28 
(a)

(b)

(c)

(d)

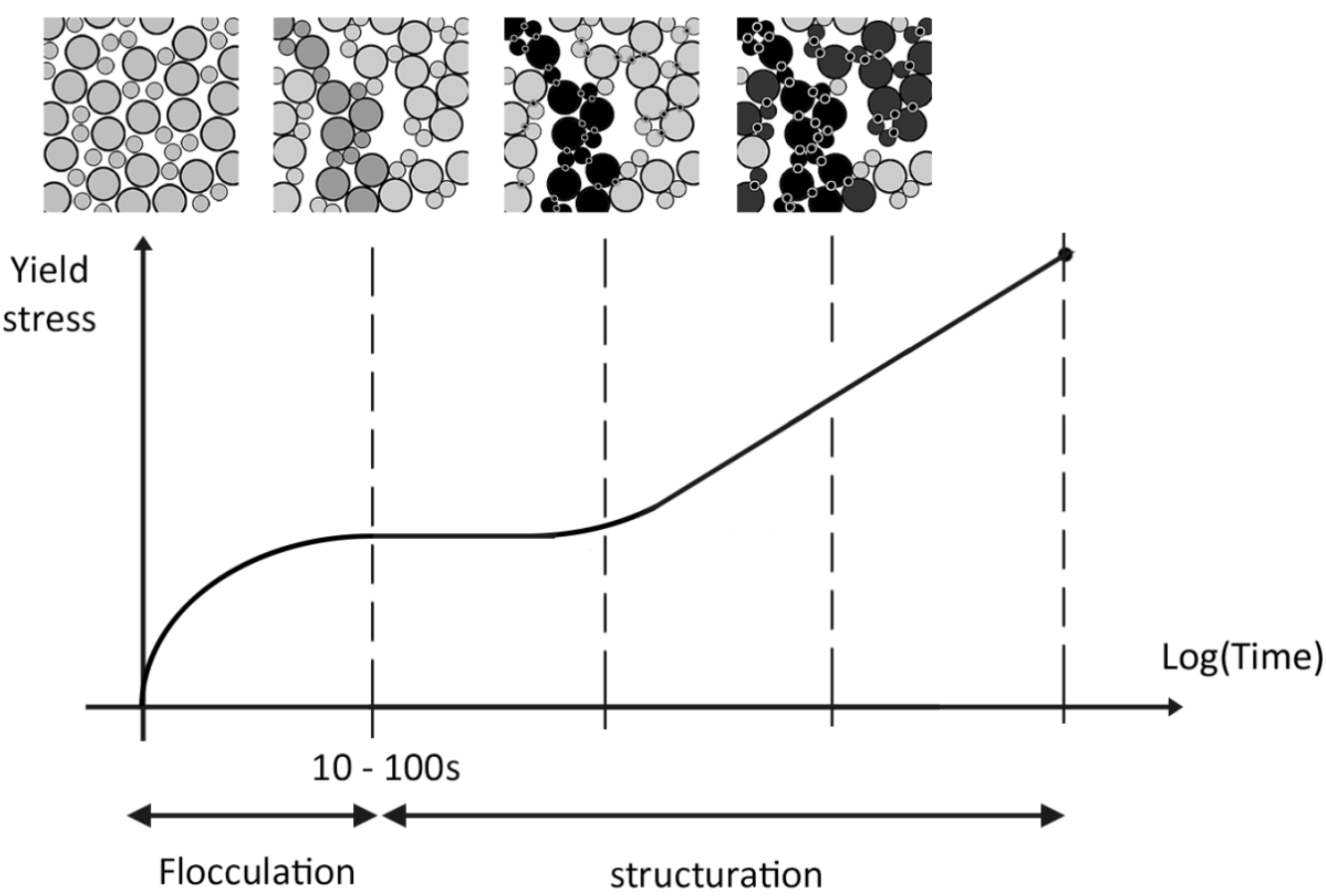

Figure 1. General behavior of fresh cement-based systems at rest described through yield stress evolution and underlying cement particle interaction and $\mathrm{C}-\mathrm{S}-\mathrm{H}$ nucleation and growth. The freshly sheared suspension starts in a dispersed state (a), then exhibits initial flocculation due to non-contact interactions. C-S-H nucleation occurs, forms bridges between cement particles/flocs (c), and gives rise to an increasingly interconnected, rigid structure (d). Reproduced from (N. Roussel et al. 2012). 


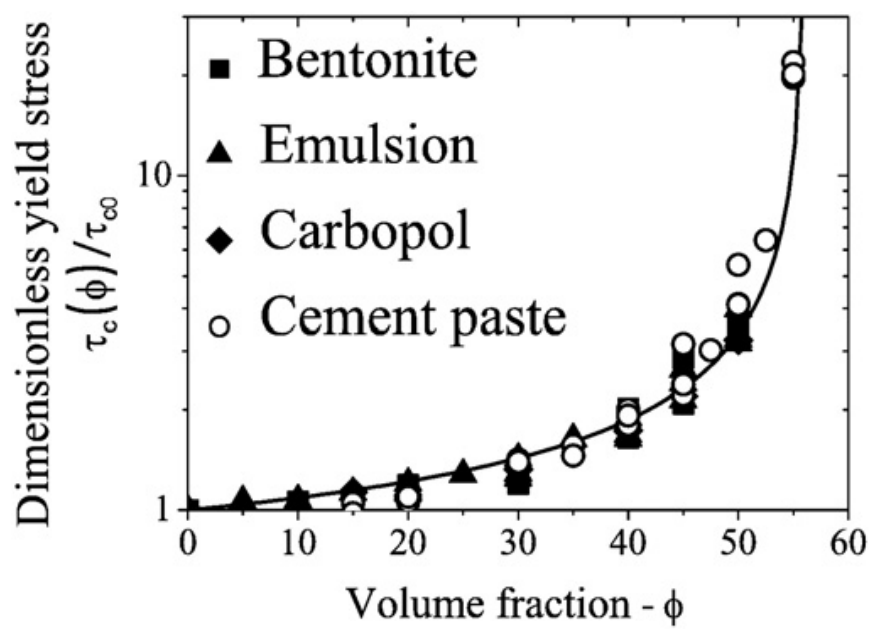

Figure 2. Dimensionless yield stress (i.e. the ratio between the yield stress of the suspension for a given volume fraction of beads and the yield tress of the suspending fluid) as a function of beads volume fraction for various suspending yield stress fluids including cement pastes (reproduced from (F. Mahaut et al., 2008b). 
a

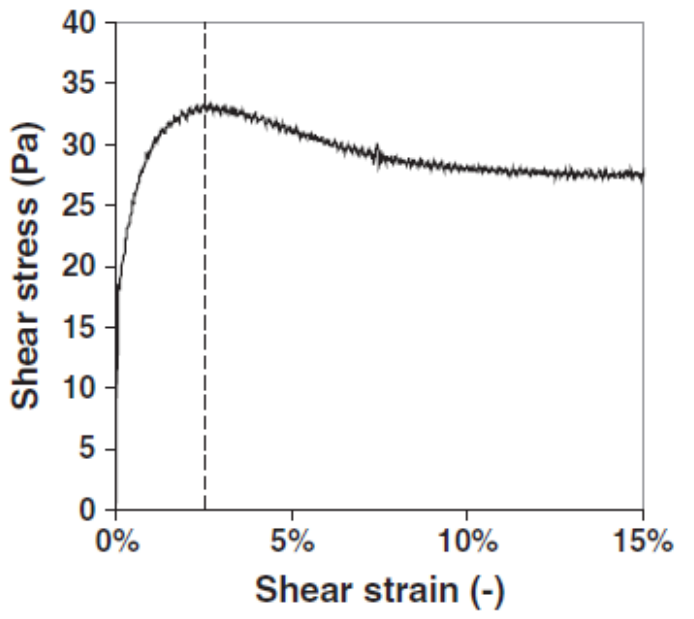

b

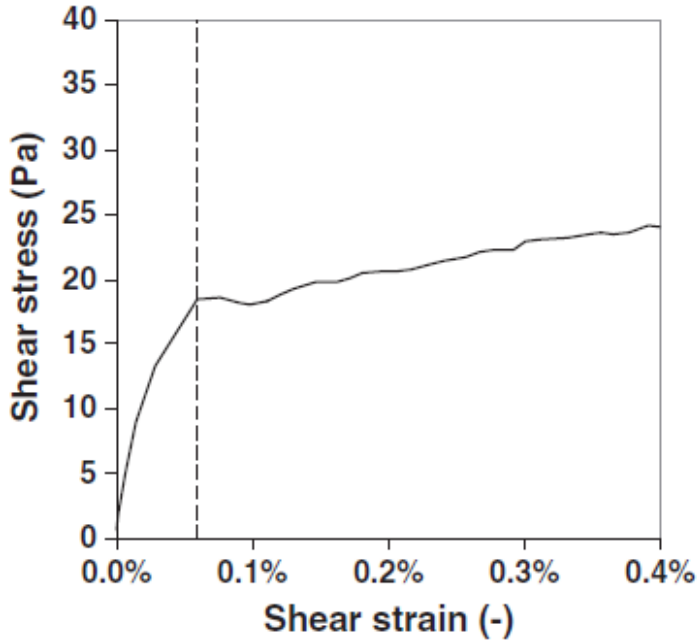

Figure 3. Shear stress as a function of shear strain during a Vane test on a cement paste with a water to cement ratio of 0.4. (a) Linear shear strain scale from 0 to 15\%; (b) Linear shear strain scale from 0 to $0.4 \%$. Extracted from [Roussel et al., 2012] 


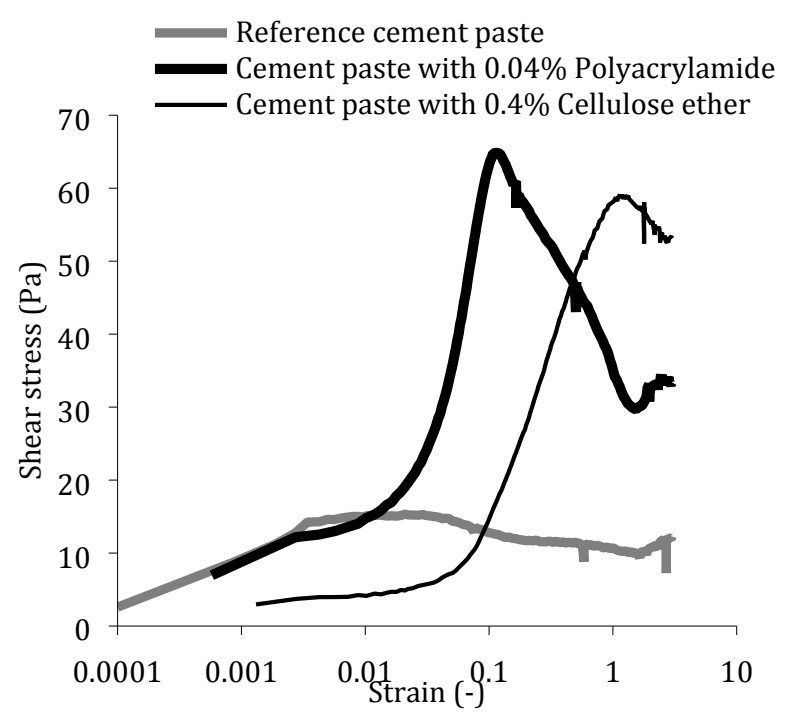

Figure 4. Flow onset measurements for a reference cement paste and pastes containing Polyacrylamide and Cellulose ether (mass dosages). 


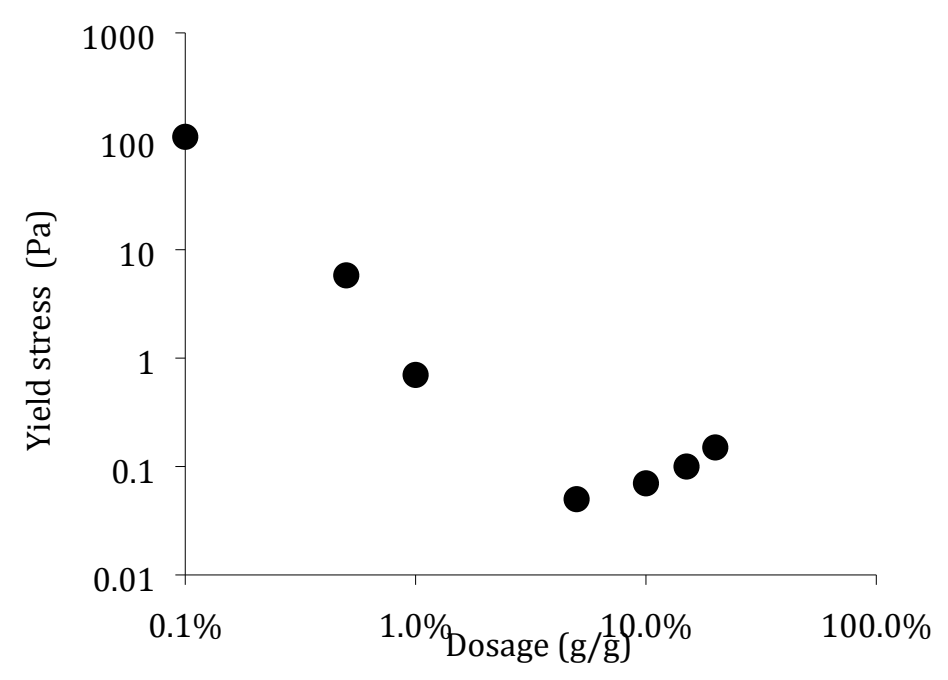

Figure 5. Yield stress of cement paste as a function of PCE dosage $(W / C=0,3)$ (extracted from $(H$. Bessaies-Bey et al., 2018)) 
--- Desorption isotherm of pre-adsorbed polycarboxylate ether

$\triangle-$ Relative yield stress of cement paste (Polycarboxylate ether + increasing dosage of sodium gluconate)

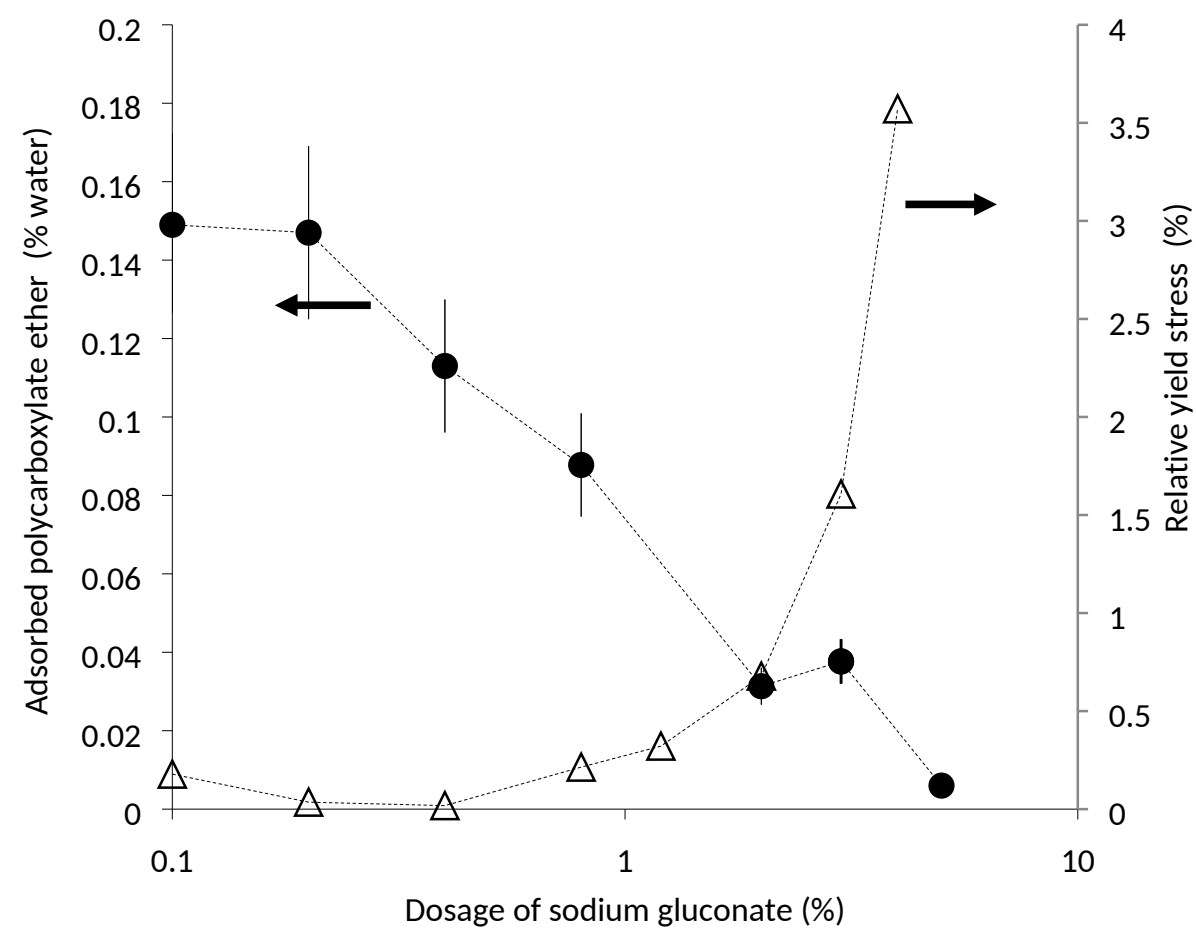

Figure 6. Desorption of a superplasticizer (polycarboxylate ether) in the presence of a retarder (sodium gluconate) (left y axis) and consequences on the yield stress (right y axis). The relative yield stress is the ratio between the yield stress of the cement paste with admixtures and the yield stress of the reference cement paste. Adapted from (H. Bessaies et al., 2016). 


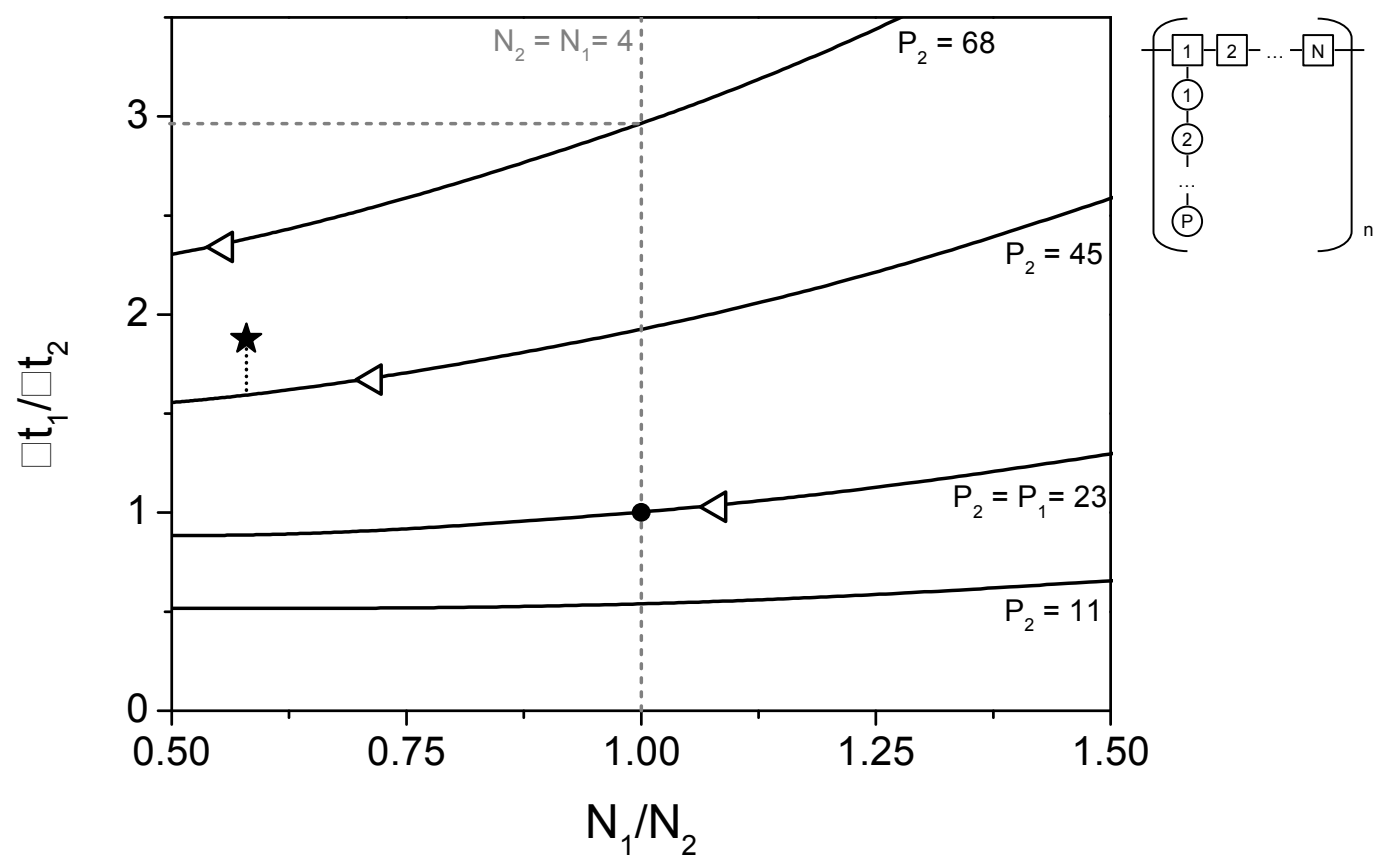

Figure 7. Abacus of retardation showing the ratios $\Delta t_{1} / \Delta t_{2}$ function of $N_{1} / N_{2}$ Ratio. $N$ is the number of monomers in the backbone for one side chain and $P$ the number of monomer in one side chain. This plot shows the evolution of the retardation as a function of changes of structural parameters with respect to a reference polymer dosed to achieve the same dispersion ability. The reference is polymer 1 with $N_{1}=4$ and $P_{1}=23$. The black dot represents the case where polymer 2 has the same structural parameters as polymer 1 . The curved lines determine the ratio of retardation $\Delta t_{1} / \Delta t_{2}$ for different side chain lengths. The vertical dashed line represents the case where $N_{1}=N_{2}$. The star represents a polymer that was used to experimentally prove that the prediction offered by this plot is realistic (reproduced from (D. Marchon et al, 2019b). 


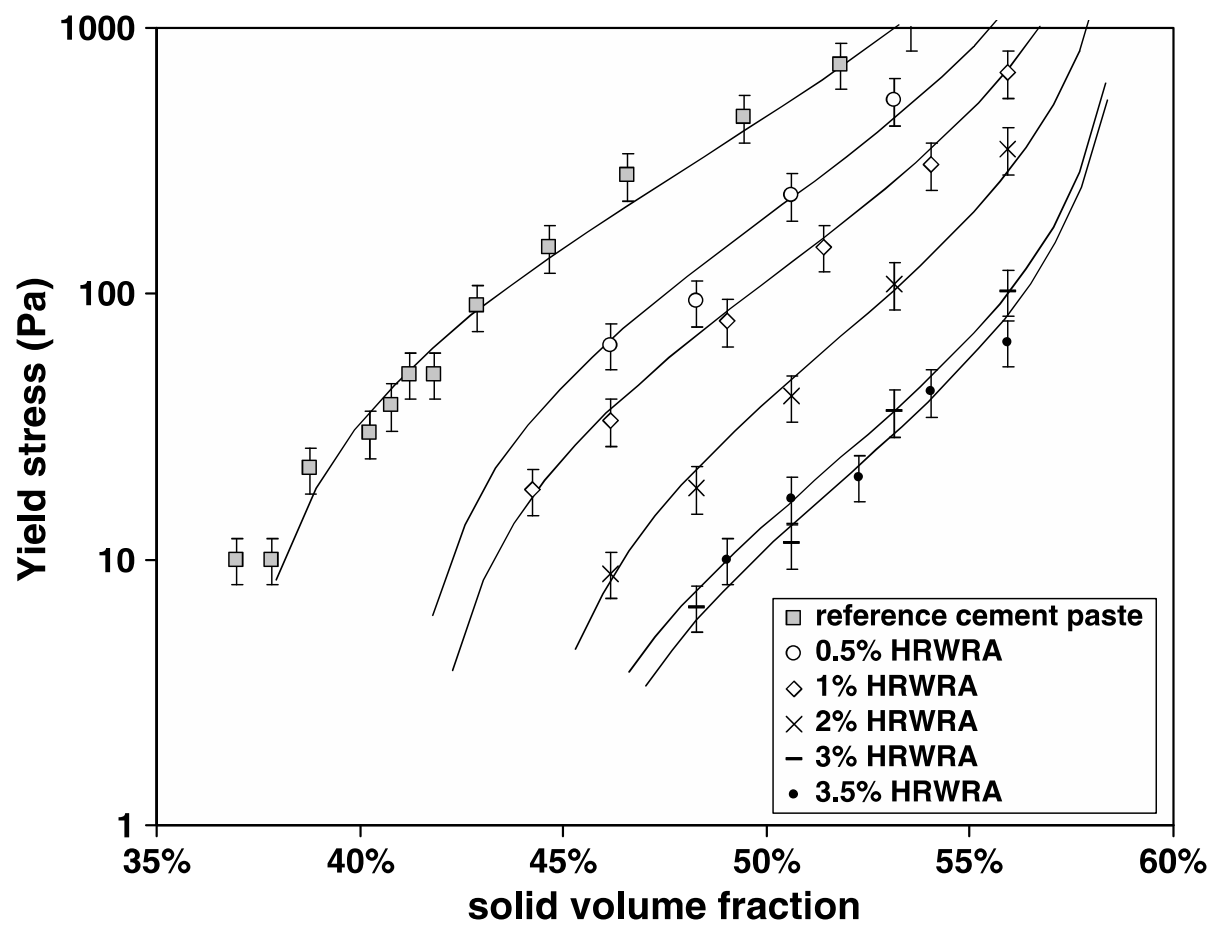

Figure 8. Yield stress as a function of cement volume fraction for various dosages of High Range Water Reducing Admixtures (HRWRA). Experimental date points along with YODEL prediction (lines). Reproduced from (A. Perrot et al., 2012). 


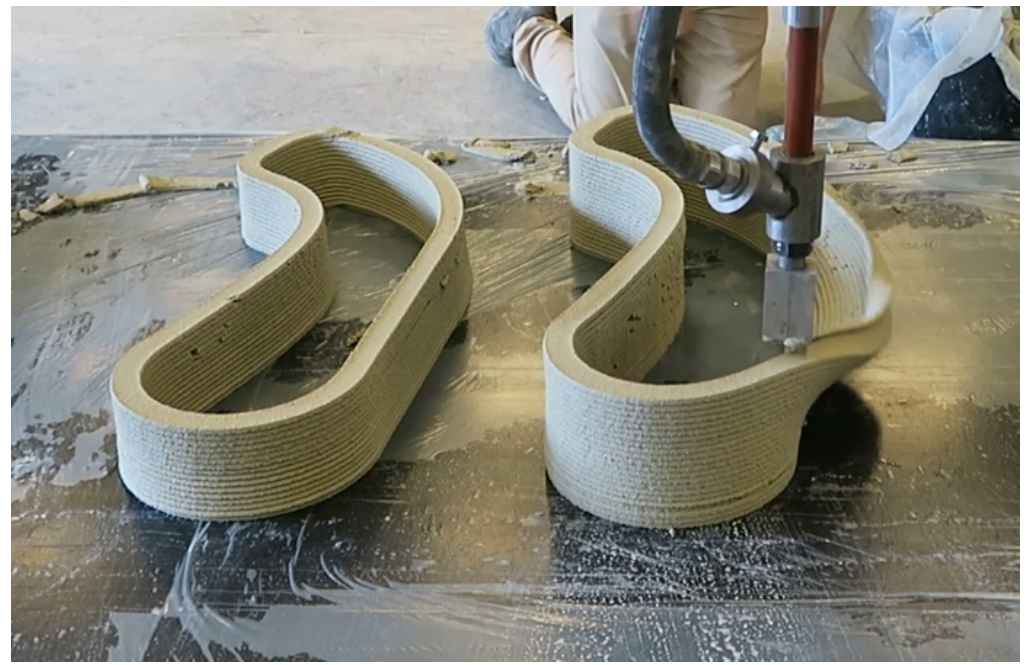

Figure 9. Elastic buckling during a 3D printing process. 